\title{
Network analysis of microRNAs, genes and their regulation in human bladder cancer
}

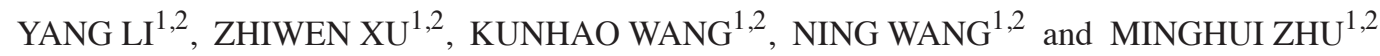 \\ ${ }^{1}$ College of Computer Science and Technology, ${ }^{2}$ Key Laboratory of Symbolic Computation and Knowledge \\ Engineering of Ministry of Education, Jilin University, Changchun, Jilin 130012, P.R. China
}

Received June 20, 2013; Accepted July 16, 2013

DOI: 10.3892/br.2013.157

\begin{abstract}
Bladder cancer (BC) is the fifth most common malignancy occurring worldwide and a significant cause of cancer-related morbidity and mortality. Although BC is a serious health issue, studies available concerning the relationship of genes, microRNAs (miRNAs) and their host genes has been lacking. In the present study, we assessed experimentally validated data from various sources that reported the effect of miRNA on various diseases, miRNA targeting of mRNAs, and combined these data with initial transcription factor (TF) binding site predictions within miRNA promoter regions. Topology networks obtained in this study included the differentially expressed, BC-associated and global networks. The three networks may be used to assess the effect of miRNAs and their regulation in human BC. By comparing and analyzing the similarities and differences among the three networks, key nodes with the largest potential of affecting the behavior of a particular network were identified. The results also showed potentially substantially influential miRNAs and TFs, which revealed subnetworks demonstrating the mechanisms involved as well as regulatory miRNA network motifs in human BC. Regulatory pathways regarding differentially expressed elements, such as genes and miRNAs, demonstrate self-adapting associations including, self-adapting associations and feedback loops in genes MYC, TP53, PTEN and 10 differentially expressed miRNAs. The differentially expressed network partially identified the BC mechanism. miRNA-targeted human BC genes were also enriched in highly relevant pathways, cell cycle regulation and apoptosis. The present study systematically delineated the pathogenesis of $\mathrm{BC}$ and provided theoretical foundations for gene therapy
\end{abstract}

Correspondence to: Professor Zhiwen $\mathrm{Xu}$, College of Computer Science and Technology, Jilin University, 2699 Qianjin Street, Changchun, Jilin 130012, P.R. China

E-mail: xuzw@jlu.edu.cn

Key words: transcription factors, microRNA, target, target genes, host gene, bladder cancer investigators to focu attention on key genes and miRNAs in future studies.

\section{Introduction}

Bladder cancer (BC) is the fifth most common malignancy occurring worldwide and a significant cause of cancer-related morbidity and mortality (1). Experimental data (2) indicated that differentially expressed genes and microRNAs (miRNAs) are crucial in the development, metastasis and therapy of BC. For example, mutations in the TP53 gene may be useful in predicting whether $\mathrm{BC}$ cells are likely to proliferate and spread to nearby tissues, and whether the disease may recur after treatment. The tumor suppressor gene PTEN, which is mutated or homozygously deleted in various types of cancer, maps to a region of $10 \mathrm{q}$ within the reported region of minimal loss in bladder tumors (3). Although there has been a focus on differentially expressed genes and miRNAs, the signaling pathway and mechanisms that elucidate miRNA transcriptional regulation remain unclear.

Transcription factors (TFs) and miRNAs are notable regulators for gene expression (4). TFs are proteins capable of activating or repressing transcription by binding to cis-regulatory elements located in the upstream regions of genes. They are capable of regulating gene expression at the transcriptional level individually or in combination with other proteins.

miRNAs are 21-24 nt regulatory, non-coding RNAs that regulate gene expression at the post-transcriptional level and are therefore important cell components. miRNAs are involved in various biological processes, including cell proliferation, differentiation and apoptosis. Calin et al (5) first confirmed that differentially expressed miRNAs are associated with BC.

miRNAs are located within genes designated as host genes. Rodriguez et al (6) suggested that miRNAs are transcribed in parallel with their host transcripts, and the two different transcription classes of miRNAs (exonic and intronic) identified in that study may require slightly different mechanisms of biogenesis. Baskerville et al (7) identified that intronic miRNA and its host gene are closely associated. Intronic miRNAs and their host genes are usually equally expressed in biological progression, usually acting as a potential partner in order to achieve biological function and affect the alteration of pathways (8). The above-mentioned results suggested 
that miRNAs together with their host gene or separately were capable of contributing to cancer progression. In this study, when miRNA was differentially expressed, we considered that the host genes were also differentially expressed and were involved in cancer progression.

The aim of the present study was to determine the associations of among genes, miRNAs and their host genes. Additionally, these transcriptional relations were regarded as a penetration point to build the regulatory network of the genes and miRNAs in human BC. The topology networks derived included the differentially expressed, BC-associated and global networks. The global network revealed experimentally validated pathways with regard to genes and miRNAs, however, this network is extremely complex and BC-associated pathways could not be determined. Therefore, we focused on the remaining two networks. Pathways regarding differentially expressed elements were extracted. The differentially expressed network partially revealed BC formation, and the topology network of BC was identified. Similarities and differences among the three networks were compared and analyzed in order to identify the key nodes and pathways. The network of differentially expressed elements partially revealed the mechanism of BC. The findings of this study may be useful for future investigations into the mechanism of action, as well as the pathogenesis and treatments of $\mathrm{BC}$.

\section{Materials and methods}

Material collection and data processing. miRNA-target interactions were extracted from TarBase 5.0 (9) and miRTarBase (10). TarBase 5.0 is a comprehensive database of experimentally supported animal miRNA targets, while miRTarBase is a database of experimentally validated miRNA-target interactions. Different databases use varying symbols to represent miRNAs and genes. In order to systemize the symbolic representation method, official symbols from the National Center for Biotechnology Information (NCBI) database, which can be accessed online at http://www.ncbi. nlm.nih.gov/gene/, were used. These experimentally validated data strongly support our study. The complete data can be considered as $\operatorname{set}^{U_{1}}$.

TF-miRNA interactions from TransmiR were extracted (11). TransmiR is a TF-miRNA regulation database. Data of TransmiR are extracted from public literature and biological experiments. The complete data of TF-miRNA interactions can be considered as $\operatorname{set}^{U_{2}}$.

miRNAs and their host genes were extracted from miRBase (12) and NCBI. miRBase provides a collection of confirmed human miRNAs. Official symbols and IDs from NCBI were used to verify host genes and their miRNAs. The complete data can be considered as set ${ }^{U_{3}}$.

Differentially expressed genes of $\mathrm{BC}$ were extracted from the KEGG pathway database (13) and the Cancer Genetics Web, which can be accessed online at: http://www.cancerindex.org. The KEGG pathway database comprises graphical diagrams of biochemical pathways as well as some of the known regulatory pathways, and can be accessed online at: http://www.kegg.com/kegg/pathway.html. For this study, the BC pathway map was employed which indicates the validated mutated BC genes. Similar methods were employed to elicit mutated BC genes from the Cancer Genetics Web. In order to make the data collected comprehensive, related SCI studies on BC-mutated genes were manually obtained. Similarly, BC-related genes including differentially expressed genes of $\mathrm{BC}$ and other genes associated with $\mathrm{BC}$ were manually obtained. In the present study, differentially expressed genes were considered as part of the related genes in the $\mathrm{BC}$-associated network. Additionally, the P-match method was used to extract TFs that may involve in BC (14). P-match is a new tool used to identify TF-binding sites in DNA sequences. It combines pattern matching and weight matrix approaches thus providing higher accuracy of recognition than each of the methods separately. TFs were regarded as BC-related genes and the focus was on the TFs set ${ }^{U_{2}}$. We downloaded 1,000 nt $(5,000 \mathrm{nt})$ promoter region sequences of targets that were targeted by mutated miRNAs from the UCSC database (15). The P-match method was used to map TF-binding sites (TFBSs) onto the promoter region of targets. P-match utilizes the matrix library as well as sets of known TF-binding sites collected in TRANSFAC, allowing the possibility to search for varying TF-binding sites (16). The vertebrate matrix and restricted high-quality criterion were used. The latter is used to indicate the matrix with a cut-off value allowing a false-negative rate of $50 \%$, while the false-positive rate is required to be reduced below a certain threshold (16). Thus, the matrices that produce the highest number of false-positive matches are defined as low-quality matrices. The complete data of differentially expressed genes and related genes can be considered as $\operatorname{set}^{U_{4}}$.

Differentially expressed miRNAs were elicited from mir2Disease (17). mir2Disease is a manually curated database that aims to provide a comprehensive resource of miRNA deregulation in various human diseases. To ensure data were comprehensive, related SCI studies on BC were manually obtained. The complete differentially expressed miRNAs and related miRNAs of BC can be considered as set ${ }^{U_{5}}$.

Three networks construction. Transcriptional network of BC is a very complex regulatory network. Differentially expressed genes and miRNAs are important in this network as they are involved in every process of cancer formation. Thus, the core regulation network of $\mathrm{BC}$ was utilized using the following method: Differentially expressed data from set ${ }_{4}$ and $\operatorname{set}^{U_{5}}$ were mapped onto set ${ }^{U_{1}}, U_{2}$ and ${ }^{U_{3}}$, then regulatory relations of TF-miRNA, miRNA-targets and hostgene-miRNA were determined. The relations were then combined to determine the core network.

Besides differentially expressed genes and miRNAs, BC-related genes and miRNAs are also involved in each process of cancer formation. Thus, a BC-related network was determined to further demonstrate the regulatory network of $\mathrm{BC}$. This network included both the core network and more complex regulatory relations. The regulation network in $\mathrm{BC}$ was obtained using the method mentioned above.

The former two networks present are crucial in regulatory relations. Besides the experimentally validated genes and miRNAs included in the former two networks, there may be some genes and miRNAs that have not been validated experimentally to be involved in the progression of $\mathrm{BC}$. In the third network, TFs and miRNAs present in the related network 


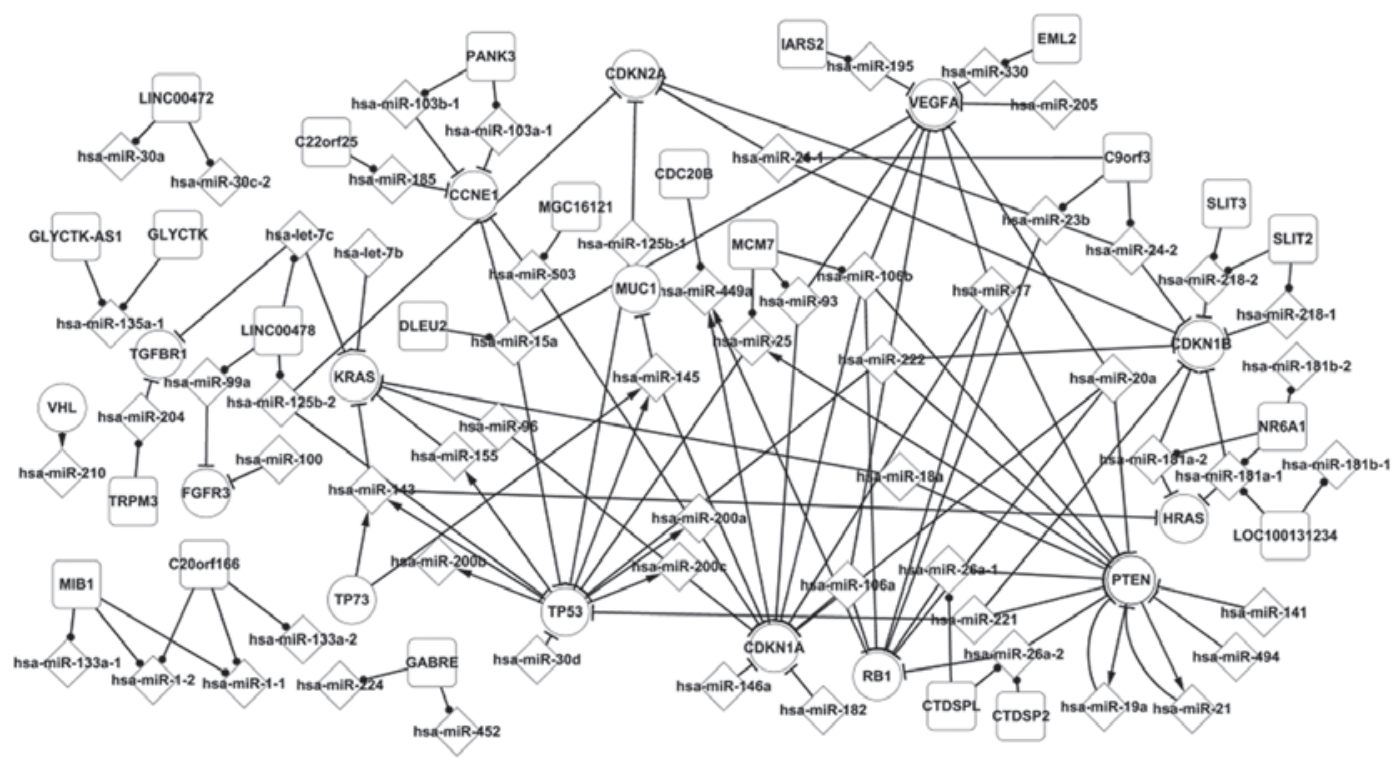

Figure 1. Core transcriptional network of differentially expressed genes, miRNAs and their host genes in bladder cancer (BC). All elements participate in various progressions of $\mathrm{BC}$, such as TP53 which participates in the cell cycle and hsa-miR-10a which contributes to the cell proliferation of BC. Relations between differentially expressed elements (genes and miRNAs) are shown in this network. They partially revealed the mechanism of BC.

were mapped onto ${ }^{U_{1}},{ }^{U_{2}}$ and ${ }^{U_{3}}$, then the regulatory relations of TF-miRNA, miRNA-targets and hostgene-miRNA were extracted. Following the combination of all relations, the expanded global network was derived.

\section{Results}

Core transcriptional network of $B C$. Through the statistical analysis, we derived a core transcriptional network that reported the regulation mechanisms in human BC. Five important TFs, i.e., TP53, TP73, CDKN1A, RB1 and PTEN, had more relations with other elements in this network and therefore were regarded as essential regulatory elements.

Fig. 1 shows some of the regulation pathways, such as PTEN which regulates hsa-miR-25a, which in turn targets TP53. TP53 regulates the miRNAs, hsa-miR-143, hsa-miR-145, hsa-miR-155, hsa-miR-200a, hsa-miR-200b and hsa-miR-200c, which target the genes KRAS, HRAS, MUC1 and CDKN1A. At the end of the pathway, hsa-miR-449a was targeted by CDKN1A. These genes and miRNAs are important elements in the progression of BC. The TP53 gene provides instructions for generating tumor protein $\mathrm{p} 53$. This protein acts as a tumor suppressor, thus it regulates cell division by keeping cells from growing and dividing too rapidly or in an uncontrolled manner. Mutations in the TP53 gene may be useful in predicting whether BC is likely to proliferate and spread to nearby tissues and whether the disease may recur following treatment. HRAS is the oncogene of BC, and it is the starting point of the MAPK signaling pathway. Mucins are a group of high molecular weight glycoproteins present on epithelial surfaces. In the bladder, they have a protective role, inhibiting bacterial and stone adhesion. Nine human mucin genes have been identified, one of which is located on chromosome 1 (18) encoding for MUC1 mucin. This mucin is upregulated (19) and abnormally glycosylated (20) in several common types of cancer, including BC (21).
The tumor suppressor gene PTEN, which is mutated or homozygously deleted in various types of cancer, maps to a region of $10 \mathrm{q}$ within the reported region of minimal loss in bladder tumors (19). PTEN is also involved in two feedback loops by targeting hsa-miR-21 and hsa-miR-19a, and vice versa.

In the present study, we utilized the core transcriptional network to explore these mechanisms of $\mathrm{BC}$.

$B C$-associated network. The related regulatory network of BC comprises differentially expressed genes and miRNAs, related genes and miRNAs, targets of miRNAs and host genes of miRNAs, with the BC-associated network including the core network. Fig. 2 shows more complex regulatory relations as compared to Fig. 1.

Fig. 1 shows six TFs, i.e., CDKN1A, RB1, PTEN, TP53, TP73, and VHL in the regulation network, which may be considered essential regulatory elements. In Fig. 2, there are 37 TFs in the regulation network, which include the six essential TFs (Fig. 1) and which regulate 59 mutated miRNAs. Among the TFs in the network BRCA1, PTEN and NFKB1 regulate more miRNA expression and therefore have a high potential of being more influential than others TFs. BRCA1 regulates hsa-miR-146a expression, which targets EGFR, NFKB1, CDKN1A and BRCA1. Thus, BRCA1 and hsa-miR-146a constitute a feedback loop.

Fig. 2 shows some additional pathways that affect the progression of $\mathrm{BC}$ with regard to differentially expressed TFs and additional miRNAs. PTEN is a mutated gene that regulates hsa-miR-19a, hsa-miR-21 and hsa-miR-25 expression. These three miRNAs target 19 relevant genes including PTEN, ESR1 and PDCD4. It has been demonstrated that ESR1 mediated a decrease in hsa-miR-21 expression correlated with an increased protein expression of endogenous hsa-miR-21 targets such as PDCD4, PTEN and BCL2 (22). Another important TF is NFKB1 which regulates the expression of 11 miRNAs including hsa-miR-21, hsa-miR-155 


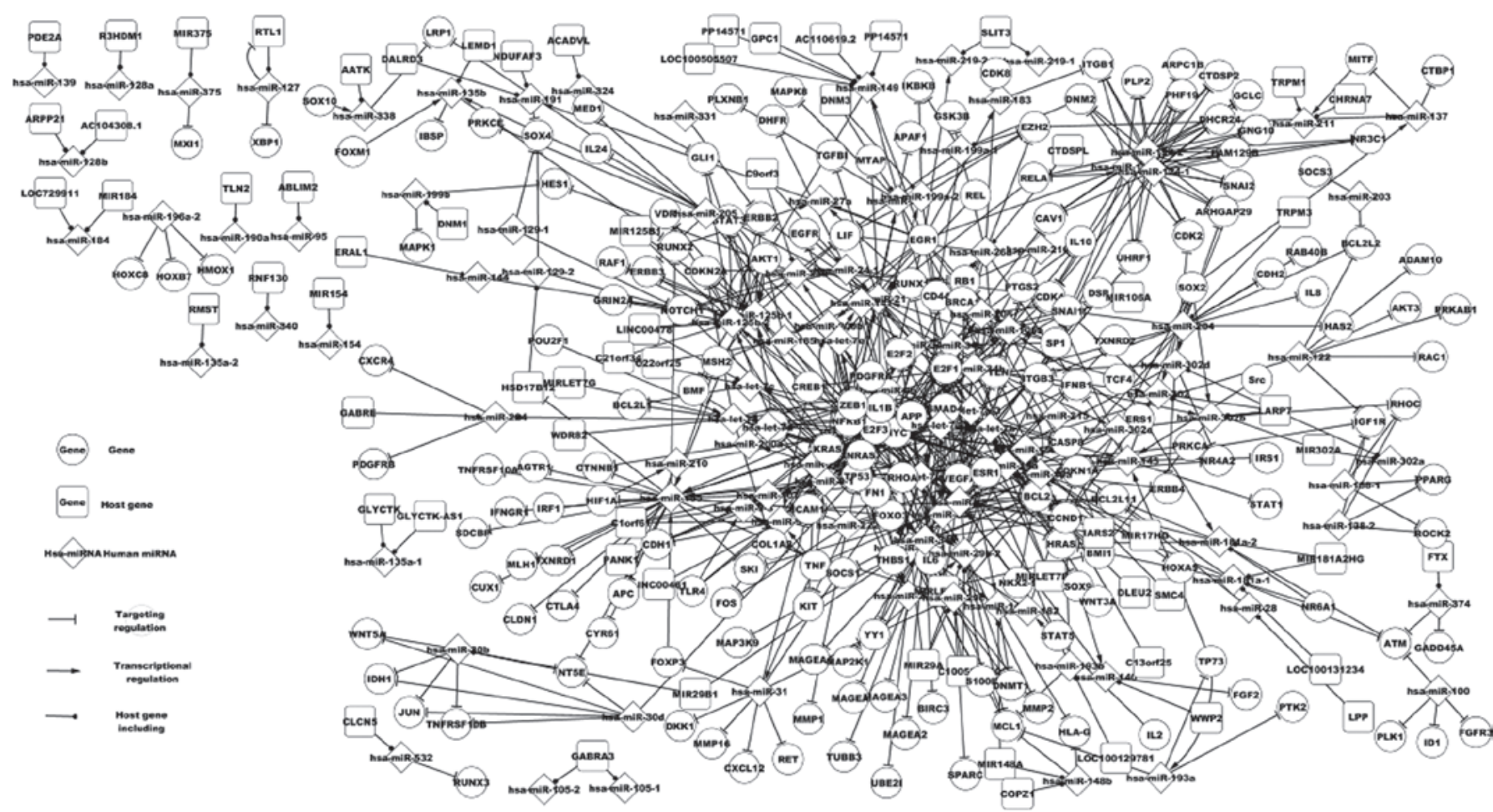

Figure 2. Bladder cancer (BC)-associated network consisting of relevant genes, miRNAs and their host genes in BC. BC-associated network expands additional topology relations of differentially expressed elements.

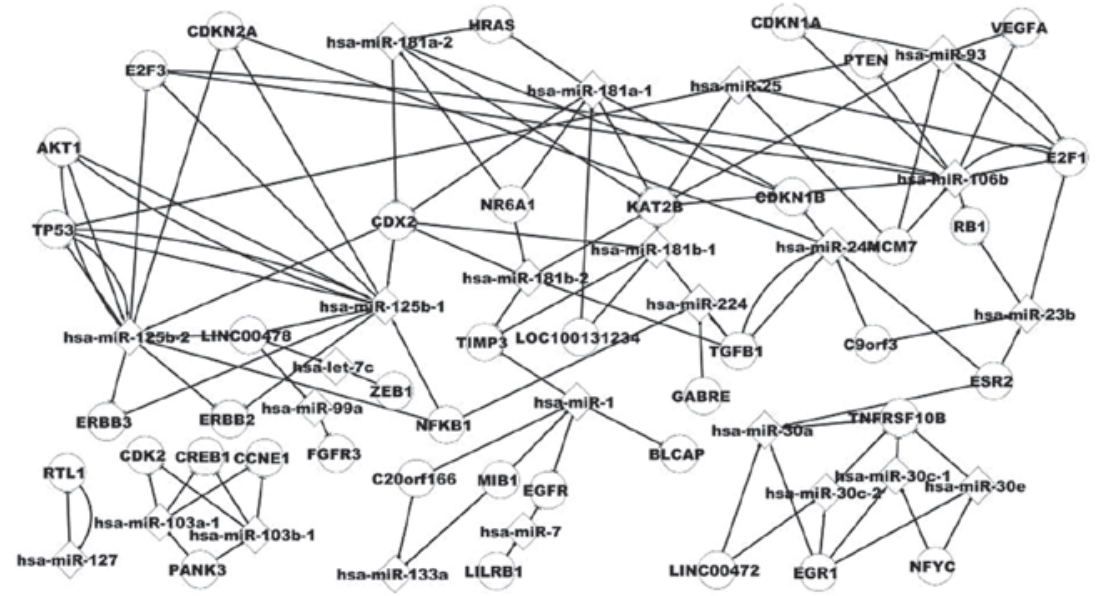

Figure 3. Specific host genes and their regulatory relations with the transcription factor, targets and their miRNAs. miRNAs can partially affect the progression to cancer together with their host gene. A host gene includes several miRNAs that target the same genes when combined or some genes when separate.

and hsa-miR-146a. Hsa-mir-155 targets 13 relevant genes, including KRAS (22).

In our network, 32 feedback loops were identified consisting of 35 genes and 32 miRNAs. Fourteen differentially expressed genes are involved in the feedback loops. Fig. 2 shows a regulation network of the interactions of 35 relevant TFs, 100 relevant miRNAs and their 161 relevant targets.

Global network of $B C$. The global regulatory network of $\mathrm{BC}$ provides more comprehensive regulatory relations. Furthermore, it has additional TFs, targets, miRNAs and host genes of miRNA compared to the related network. The global network includes more comprehensive regulatory relations including all relations in ${ }^{U_{1}},{ }_{2}$ and ${ }^{U_{3}}$. This network includes both the differentially expressed and BC-associated network.

Analysis of the host gene and its miRNA in BC. The host gene and its miRNA demonstrate some important features in this study. Alhough these host genes are not differentially expressed genes of $\mathrm{BC}$, we considered them as differentially expressed genes when miRNAs they regulate are differentially expressed miRNAs. Fig. 3 shows some pathways of host genes and miRNAs, for example, NR6A1 includes hsa-miR-181a-2, which targets HRAS. The association between hsa-miR-93 and E2F1 is noteworthy. E2F1 includes hsa-miR-93 and is targeted by hsa-miR-93. A host gene includes several miRNAs, 
Table I. Regulatory relation between microRNAs (miRNA) and PTEN.

\section{PTEN}

miRNAs that target genes

\begin{tabular}{lcc}
\hline $\begin{array}{l}\text { Differentially } \\
\text { expressed miRNAs }\end{array}$ & Related miRNAs & Global \\
miRNAs
\end{tabular}

miRNAs that are regulated by genes

Differentially

expressed miRNAs Related miRNAs Global miRNAs

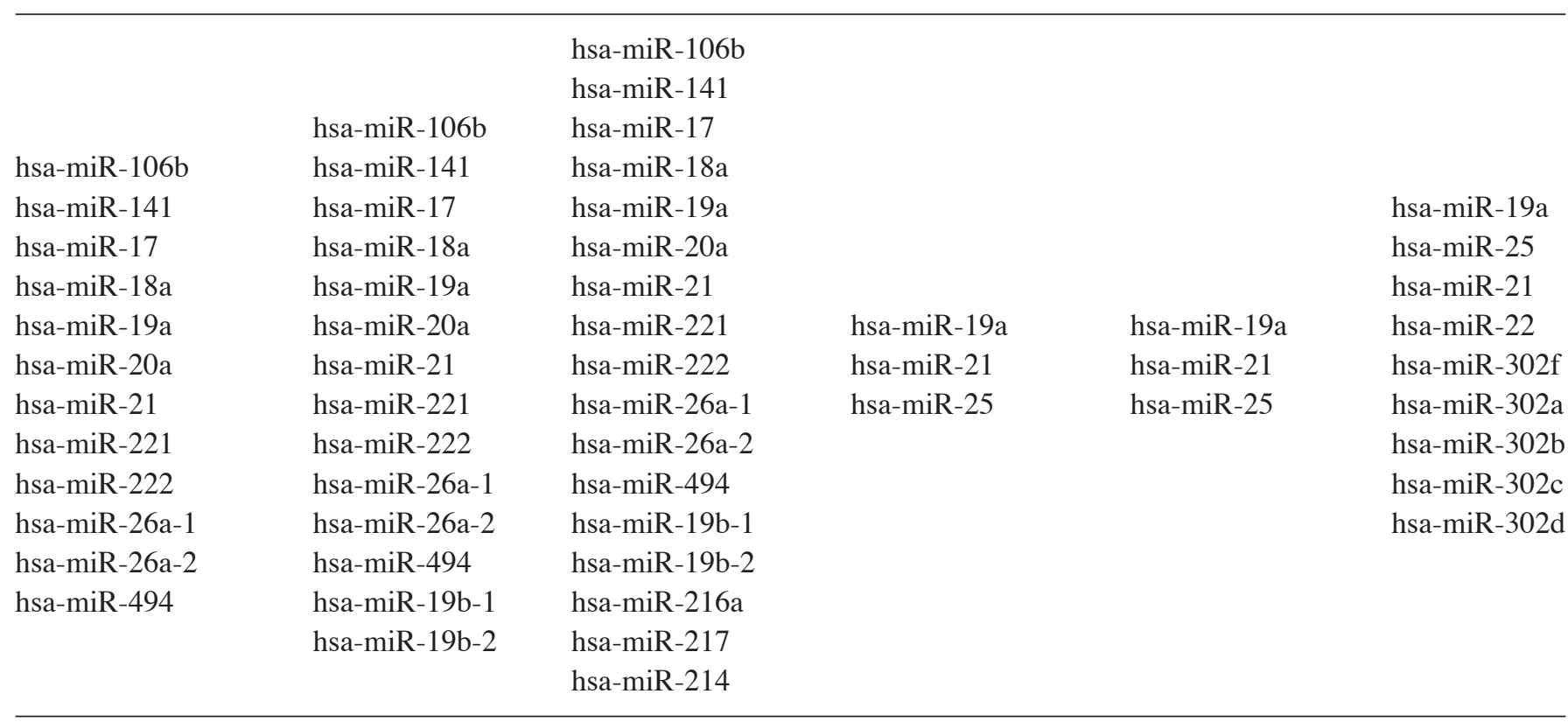

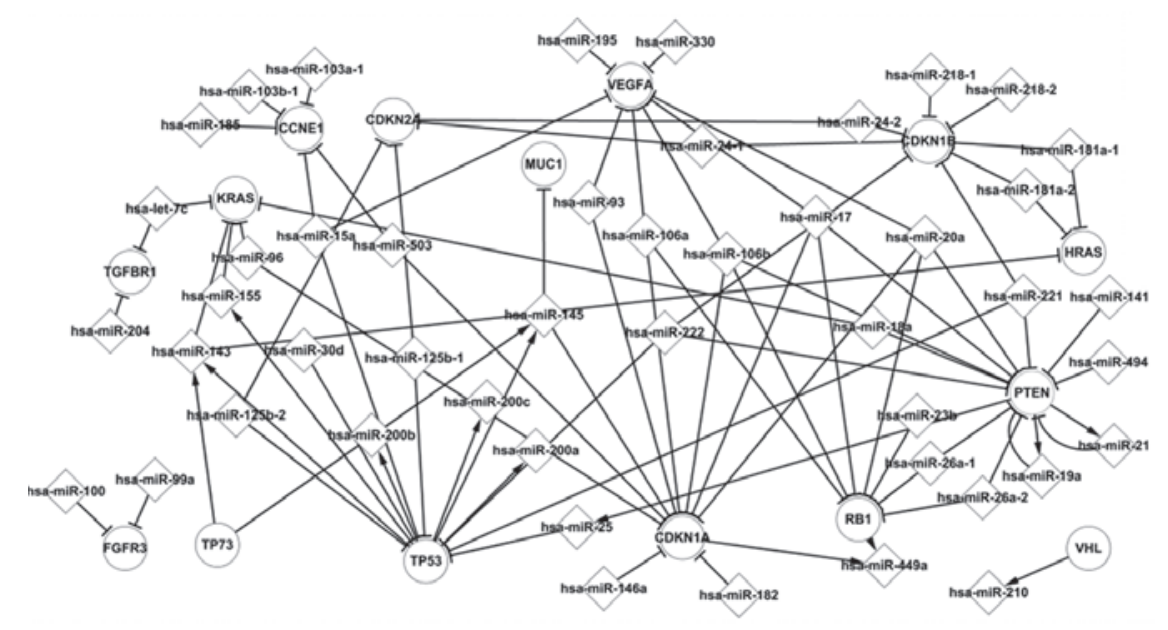

Figure 4. Transcriptional network of popular transcription factors (TFs) and differentially expressed microRNAs (miRNAs). This figure shows that popular TFs regulate differentially expressed miRNAs which target differentially expressed genes. These popular TFs are frequently involved in the transcriptional progression of cancer.

for example, MIB1 includes hsa-miR-1 and hsa-miR-133a. A miRNA is located in several genes, for example, hsa-miR-133a includes the host genes MIB1 and C20orf166.

It is therefore suggested that host genes and their miRNAs may be useful in our understanding of the pathogenesis of BC.

Transcriptional network of TFs and differentially expressed miRNAs. There are 82 miRNAs in the differentially expressed network, of which 47 are included in Fig. 4. These miRNAs and popular TFs constitute a transcriptional network. They present some significant characteristics in BC formation. TFs and miRNAs present several types of regulatory relations and affect the expression of their target elements. Fig. 4 shows that PTEN regulates three miRNAs, while being targeted by 12 miRNAs. These miRNAs, i.e., hsa-miR-19a, hsa-miR-21 and hsa-miR-25, are regulated by CDKN1A, but also target CDKN1A. CDKN1A regulates hsa-miR-25, which in turn targets TP53. TP53, RB1, TP73 and VHL are similar to CDKN1A and together with differentially expressed miRNAs interact with each other to influence the progression of $\mathrm{BC}$. 
Fig. 4 also shows that one differentially expressed miRNA is regulated by several TFs, one TF is targeted by several differentially expressed miRNAs, one TF indirectly influenced another TF by differentially expressed miRNA and one differentially expressed miRNA indirectly influenced another miRNA by TF.

Regulatory pathways of differentially expressed genes. To describe the BC network more clearly, we extracted the upstream and downstream information of the important elements: differentially expressed genes, differentially expressed miRNAs and popular TFs from the P-match method.

Nodes were classified according to the regulatory relations, with adjacent nodes for the three-level networks in order to compare and analyze the interactions of each differentially expressed gene. Of the genes investigated, CDKN1A, PTEN, TP53 and RB1 demonstrated the special feature involving each gene regulating miRNA(s) and being regulated by the $\operatorname{miRNA}(\mathrm{s})$.

\section{Discussion}

In the present study, we initially focused on TFs. The first class of TF has six types of adjacent nodes (three types of successors and three types of predecessors). This class of TFs includes CDKN1A, PTEN, TP53 and RB1.

Table I provides evidence of the relationship of PTEN and miRNAs in each network. In the core network, 12 miRNAs targeting PTEN were identified, with PTEN regulating three miRNAs. In the BC-associated network 14 miRNAs (2 additional miRNAs: hsa-miR-19b-1 and hsa-miR-19b-2) were identified to targetPTEN, with PTEN regulating three miRNAs. In the global network, 17 miRNAs (3 additional miRNAs: hsa-miR-214, hsa-miR-217 and hsa-miR-216a) were identified to target PTEN, with PTEN regulating 9 miRNAs (6 additional miRNAs: hsa-miR-22, hsa-miR-302a, hsa-miR-302b, hsa-miR-302c, hsa-miR-302d and hsa-miR-302f). These predecessors indirectly affected the successors through PTEN. Of all the miRNAs, we observed that hsa-miR-19a targets PTEN and PTEN regulates hsa-miR-19a in all three networks. Additionally, they form self-adapting associations and are both differentially expressed elements in BC.

The second class of TFs has five types of adjacent nodes (two types of successor and three types of predecessor), for example, KRAS. Two differentially expressed miRNAs target KRAS, however, KRAS does not regulate any miRNA.

The third class of TFs has four types of adjacent nodes (three types of successors and a type of predecessor, a type of successor and three types of predecessors), such as CDKN1A. Four miRNAs target CDKN1A, which, however, does not regulate any miRNA in the differentially expressed or BC-associated networks.

The fourth class of TFs has three types of adjacent nodes (three types of successors), for example VHL. No miRNA targets VHL, which only regulates hsa-miR-210.

We also focused on the genes that do not regulate any miRNA. The first class of genes has three types of adjacent nodes (three types of predecessors), including KRAS and HRAS. Although they are targeted by some miRNAs, they do not regulate any miRNA, possibly due to their being the last node in the pathway. Oncogenic HRAS directly results in the development of BC (18).

The second class of genes has a type of adjacent node (a type of predecessor), for example GRB2. GRB2 is targeted by two miRNAs in the global network but does not regulate any miRNA. It is suggested that GRB2 has the least influence compared to any other differentially expressed genes.

In conclusion, we collected all currently validated genes and miRNAs associated with BC and derived three regulatory networks to analyze complex regulatory relations of differentially expressed elements in BC. We extracted and compared the similarities and differences of all differentially expressed elements in the three networks to distinguish the key nodes and pathways that contribute to understanding the tumorigenic mechanism and therapy of BC. We observed that some pathways of differentially expressed elements have been validated in $\mathrm{BC}$, while other pathways have not been validated in $\mathrm{BC}$ although they have an impact on other types of cancer. Additionally, some related pathways in the $\mathrm{BC}$-associated network and additional pathways in the global network also affect the progression of other types of cancer. Pathways of differentially expressed elements must be involved in $\mathrm{BC}$, however, the majority of their mechanisms remain unclear. Consequently, these pathways which have not been validated in BC potentially affect the progression of cancer. However, their exact role in $\mathrm{BC}$ remains to be clarified and additional investigations on these pathways in BC should be conducted. The present study has provided comprehensive data associated with $\mathrm{BC}$ which is useful in investigations regarding the mechanism of differentially expressed genes and miRNAs in BC. In the subsequent studies, transcriptional co-factors and the interaction of proteins in our network are to be further investigated in order to derive a more comprehensive and extensive network to obtain greater insights into the pathogenesis and treatments of BC.

\section{Acknowledgements}

This study was supported by grants from the National Natural Science Foundation of China (grant nos. 60973091 and 60905022).

\section{References}

1. Jemal A, Murray T, Ward E, et al: Cancer statistics, 2005. CA Cancer J Clin 55: 10-30, 2005.

2. Pashos CL, Botteman MF, et al: Bladder cancer: epidemiology, diagnosis, and management. Cancer Pract 10: 311-322, 2002.

3. Wang DS, Rieger-Christ K, Latini JM, et al: Molecular analysis of PTEN and MXI1 in primary bladder carcinoma. Int J Cancer 88: 620-625, 2000.

4. Hobert O: Gene regulation by transcription factors and microRNAs. Science 319: 1785-1786, 2008.

5. Calin GA, Dumitru CD, Shimizu M, et al: Frequent deletions and down-regulation of micro-RNA genes miR15 and miR16 at 13q14 in chronic lymphocytic leukemia. Proc Natl Acad Sci USA 99: 15524-15529, 2002.

6. Rodriguez A, Griffiths-Jones S, Ashurst JL and Bradley A: Identification of mammalian microRNA host genes and transcription units. Genome Res 14: 1902-1910, 2004.

7. Baskerville S and Bartel DP: Microarray profiling of microRNAs reveals frequent coexpression with neighboring miRNAs and host genes. RNA 11: 241-247, 2005.

8. Cao GJ, Huang BC, Liu ZH, et al: Intronic miR-301 feedback regulates its host gene, ska2, in A549 cells by targeting MEOX2 to affect ERK/CREB pathways. Biochem Biophys Res Commun 396: 978-982, 2010. 
9. Sethupathy P, Corda B and Hatzigeorgiou AG: TarBase: a comprehensive database of experimentally supported animal microRNA targets. RNA 12: 192-197, 2006.

10. Hsu SD, Lin FM, Wu WY, et al: miRTarBase: a database curates experimentally validated microRNA-target interactions. Nucleic Acids Res 39: D163-D169, 2011.

11. Wang J, Lu M, Qiu CX and Cui QH: TransmiR: a transcription factor-microRNA regulation database. Nucleic Acids Res 38 D119-D122, 2010

12. Griffiths-Jones S, Saini HK, van Dongen S and Enright AJ: miRBase: tools for microRNA genomics. Nucleic Acids Res 36 D154-D158, 2008

13. Kanehisa M, Goto S, Furumichi M, Tanabe M and Hirakawa M: KEGG for representation and analysis of molecular networks involving diseases and drugs. Nucleic Acids Res 38: D355-D360, 2010.

14. Chekmenev DS, Haid C and Kel AE: P-Match: transcription factor binding site search by combining patterns and weight matrices. Nucleic Acids Res 33: W432-W437, 2005.

15. Dreszer TR, Karolchik D, Zweig AS, et al: The UCSC Genome Browser database: extensions and updates 2011. Nucleic Acids Res 40: D918-D923, 2012.

16. Kao A, Poteet SR, Jones DH et al: P-MATCH: Identifying Part Name in Noisy Text Data. In: Cross-Disciplinary Advances in Applied Natural Language Processing: Issues and Approaches. Boonthum-Denecke C, McCarthy PM and Lamkin T (eds). IGI Global, Hershey, pp172-184, 2011.
17. Jiang QH, Wang YD, Hao YY, et al: miR2Disease: a manually curated database for microRNA deregulation in human disease. Nucleic Acids Res 37: D98-D104, 2009.

18. Swallows DM, Gendler S, Gribths B, et al: The hypervariable gene locus PUM, which encodes for the tumour associated epithelial mucins, is located on chromosome 1, within the region 1q21-24. Ann Hum Genet 51: 289-294, 1987.

19. Zotter S, Hageman PC, Lossnitzer A, Mooi WJ and Hilgers J: Tissue and tumour distribution of human polymorphicepithelial mucin. Cancer Rev 11: 55-79, 1988.

20. Girling A, Bartkova J, Burchell J, et al: A core protein epitope of the polymorphic epithelial mucin detected by the monoclonal antibody SM-3 is selectively exposed in a range of primary carcinomas. Int J Cancer 43: 1072-1076, 1989.

21. Simms MS, Hughes ODM, Limb M, Price MR and Bishop MC: MUC1 mucin as a tumour marker in bladder cancer. BJU Int 84: 350-352, 1999.

22. Kompier LC, Lurkin I, van der Aa MN, van Rhijn BW, van der Kwast TH and Zwarthoff EC: FGFR3, HRAS, KRAS, NRAS and PIK3CA mutations in bladder cancer and their potential as biomarkers for surveillance and therapy. PloS One 5: e13821, 2010. 